\title{
Oncological outcome of patients with salivary gland cancer treated with surgery and postoperative intensity-modulated radiotherapy: a retrospective study
}

\section{Shoumei Zang}

Zhejiang University School of Medicine First Affiliated Hospital

Huijie Huang

Yili Friendship Hospital

\section{Xinli Zhu}

Zhejiang University School of Medicine First Affiliated Hospital

\section{Meiqin Chen}

Zhejiang University School of Medicine First Affiliated Hospital

\section{Danfang Yan}

Zhejiang University School of Medicine First Affiliated Hospital

Senxiang Yan ( $\square$ yansenxiang@zju.edu.cn )

Zhejiang University School of Medicine First Affiliated Hospital

\section{Research}

Keywords: salivary gland cancer, IMRT, survival

Posted Date: March 4th, 2020

DOI: https://doi.org/10.21203/rs.3.rs-15982/v1

License: (c) (i) This work is licensed under a Creative Commons Attribution 4.0 International License.

Read Full License

Version of Record: A version of this preprint was published at Quantitative Imaging in Medicine and Surgery on January 1st, 2021. See the published version at https://doi.org/10.21037/qims-21-836. 


\section{Abstract}

Background To investigate the outcomes, prognostic factors, patterns of failure, and adverse events in patients with salivary gland cancer (SGC) treated with surgery and postoperative intensity-modulated radiotherapy (IMRT).

Methods We identified 60 patients with major SGC treated with surgery followed by postoperative IMRT. Data for overall survival (OS),progression-free survival (PFS), locoregional relapse-free survival (LRRFS), distant metastasis-free survival (DMFS), prognostic factors, and treatment-related toxicities were analyzed. Survival was calculated with the Kaplan-Meier method. Multivariable analysis (MVA) was used to identify prognostic factors for OS, PFS, LRRFS and DMFS.

Results Adenoid cystic carcinoma (ACC) was the most common histology ( $n=21 ; 35 \%$ ). With a median follow-up of 55.5 months, OS and PFS were $90.7 \%, 85.1 \%$, and $85.1 \%$; and $80.1 \%, 72.7 \%$, and $63.1 \%$, at 3 , 5 , and 10 years, respectively. LRRFS and DMFS at 3,5 , and 10 years were $87.4 \%, 82.1 \%$, and $82.1 \%$; and $85.3 \%, 78.4 \%$, and $66.1 \%$, respectively. Five-year OS, PFS, LRRFS, and DMFS for ACC was $100 \%, 67.7 \%$, $76.2 \%$, and $90.2 \%$, respectively. In MVA, N stage was an independent predictor of PFS ( $p=0.047)$. Positive margin was a significant prognostic factor for PFS, LRRFS, and DMFS ( $p=0.036,0.026$, and 0.011 , respectively). Major nerve involvement was significantly correlated with PFS and DMFS ( $p=0.034$ and 0.008 , respectively). Interval from surgery to radiotherapy (RT) predicted PFS and DMFS ( $p=0.036$ and 0.012 , respectively). The most common acute toxicities were mucositis and dermatitis, and xerostomia was the most common late adverse event. Lung metastasis was the most common pattern of distant failure.

Conclusion Postoperative IMRT leads to improved survival for SGC patients with acceptable toxicities.

\section{Introduction}

Salivary gland cancer (SGC) constitutes a heterogeneous group of diseases, accounting for only $3-6 \%$ of all head and neck cancer [1]. Although its etiology remains unclear, SGC prognosis has improved thanks to combined treaments of surgery, postoperative radiotherapy (PORT) and chemotherapy. Retrospective reviews have shown that prognosis for SGC patients depends on SGC histology, grade, and stage [2-4]. Other characteristics affecting prognosis include positive margin, extracapsular extension, bone and perineural invasions [1].

However, it is difficult to compare studies owing to differences in the number of patients enrolled in each study, pathological types, and treatment strategies. Intensity-modulated radiotherapy (IMRT) has led to increased treatment accuracy and the possibility of delivering higher doses to the tumor region. This suggests that previous studies that included patients who were irradiated using conventional radiotherapy (RT) or 3-dimensional conformal radiotherapy (3-DCRT) may have yielded lower locoregional control rates. We focused on postoperative IMRT, which was developed to improve local tumor control rate and quality of life and has been widely adopted for the treatment of head and neck 
cancer in recent decades. Because of the differences in biological behavior between major and minor SGC, prognosis varies considerably. Here, we collected and analyzed comprehensive treatment outcomes for major SGC patients treated with surgery and postoperative IMRT, and explored survival, related adverse prognostic factors, treatment failure patterns, and adverse events.

\section{Materials And Methods}

\section{Patients}

We retrospectively reviewed 60 patients with histologically confirmed primary SGC treated at the First Affiliated Hospital of Zhejiang University from January 2009 to December 2016. All the patients had newly diagnosed diseases and received upfront surgery followed by external beam RT using IMRT. Patients with recurrent or metastatic disease or those who failed to finish the scheduled RT were excluded.

\section{Evaluation}

All patients were initially evaluated by a multimodality treatment team consisting of an otolaryngologist, a medical oncologist, and a RT oncologist. All patients underwent a detailed physical examination. Histological confirmation of SGC was required before treatment. Axial imaging with computed tomography (CT) was a routine part of patient evaluation, and most patients had also undergone either magnetic resonance imaging (MRI) or positron emission tomography (PET). Histological diagnosis was confirmed according to the WHO histologic subtype criteria for SGC [5]. All patients were restaged based on the 2018 American Joint Committee on Cancer classification. Acute adverse events were graded according to electronic records. Late toxicity was assessed based on follow-up visits.

\section{Treatment}

All patients underwent initial primary resection, with neck dissection (ND) therapeutically (if clinically positive lymph node; $\mathrm{CN}+$ ) or electively in high-risk $\mathrm{cNO}$ (clinically negative lymph node) patients. For IMRT, patients were immobilized in the supine position with a thermoplastic head-neck or head-neckshoulder mask to ensure the daily reproducibility of treatments. Simulation CT scan was performed with 3-mm slice thickness and transported to the treatment planning system. Clinical target volume (CTV) was defined as postoperative tumor bed and the elective nodal area (Figure 1). Elective nodal irradiation target volume comprised the positive lymph nodal areas plus at least one level beyond. Organ-at-risk volumes (spinal cord, optic apparatus, mandible) were delineated on each slice. The maximal dose constraints were below $45 \mathrm{~Gy}$ for the spinal cord, $55 \mathrm{~Gy}$ for the optic apparatus, and $70 \mathrm{~Gy}$ for the mandible. For the planning target volume (PTV), a 0.3-cm margin was applied to the CTV considering daily setup error. The prescribed dose was 60-68 Gy, administered at a daily $2 \mathrm{~Gy} /$ fraction, 5 days/week over 6-6.8 weeks (30-34 fractions). 
Chemotherapy was administered to patients with advanced disease or in the presence of high pathological risk factors. Cisplatin ( $80 \mathrm{mg} / \mathrm{m}^{2}$ intravenously every 3 weeks) was the most commonly used concomitant chemotherapy schedule. Several patients received adjuvant chemotherapy of cisplatin (80 mg/m² intravenously, day 1$)$ and $5-\mathrm{FU}\left(1000 \mathrm{mg} / \mathrm{m}^{2}\right.$ continuous infusion over $\left.120 \mathrm{~h}\right)$, or cisplatin (80

$\mathrm{mg} / \mathrm{m}^{2}$ intravenously, day 1$)$ and capecitabine $\left(1250 \mathrm{mg} / \mathrm{m}^{2}\right.$ orally twice a day, day 1 to day 14$)$ repeated every 3 weeks followed by PORT.

\section{Follow-up}

After treatment completion, patients were evaluated every 3 months for the first 1 year, every 3-6 months over the following 4 years, and yearly thereafter. At each follow-up visit, a physical examination and imaging were performed, including fiberoptic endoscopy if indicated. A PET scan was performed if recurrence or metastasis was suspected. Overall survival (OS) was defined as the time from surgery to the date of death; progression-free survival (PFS) as the time from surgery to the date of local or regional recurrence, distant metastases, or death from any cause; locoregional relapse-free survival (LRRFS) as absence of disease recurrence in the local site or regional lymph node; and distant metastasis-free survival (DMFS) as the time to distant metastasis.

\section{Statistical analysis}

Survival was analyzed using the Kaplan-Meier method and compared using the log-rank test. A Cox proportional hazards regression model was used for MVA. $p<0.05$ was considered significant. Statistical tests were two-sided. All data were analyzed using SPSS 20.0.

\section{Results}

\section{Clinicopathological characteristics}

Patient characteristics are summarized in Table 1. The median follow-up was 55.5 months (range 1-114 months). Median age at initial diagnosis was 52 years (range 15-76 years). Among the patients, 35 (58.3\%) were male and 25 (41.7\%) female. Median tumor size was $2.5 \mathrm{~cm}$ (range $1-15 \mathrm{~cm}$ ). Most patients presented with a palpable mass at initial presentation. Twenty-three (38.3\%) experienced pain and $5(8.3 \%)$ showed facial nerve paralysis. In some patients, painless masses had remained stable for several years and then began to grow rapidly. Histologic types included ACC in 21 patients (35\%), lymphoepithelial carcinoma (LELC) in 12 (20\%), mucoepidermoid carcinoma (MEC) in 8 (13.3\%), salivary duct carcinoma (SDC) in 5 (8.3\%), squamous cell carcinoma (SCC) in $4(6.7 \%)$, basal cell adenocarcinoma (BCAC) in $3(5 \%)$, myoepithelial carcinoma in $2(3.3 \%)$, acinic cell carcinoma (AcCC) in 1 $(1.7 \%)$, and others in $4(6.7 \%)$. The primary sites were the parotid gland in 34 patients $(56.7 \%)$, submandibular gland in $17(28.3 \%)$, and sublingual gland in $9(15 \%)$. The T stage distribution was 14 (23.3\%) T1-T2 and 46 (76.7\%) T3-T4b. The N stage distribution was 41 (68.3\%) N0, 8 (13.3\%) N1, 8

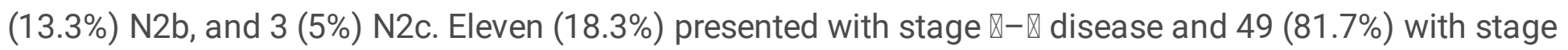
$\bigotimes$ - $₫$ b. Skin involvement was observed in 5 patients (8.3\%), positive margin in $7(11.7 \%)$, extra- 
parenchymal extension in 34 (56.7\%), perineural invasion in 19 (31.7\%), and major nerve involvement in $28(46.7 \%)$.

\section{Treatment characteristics}

All patients received primary tumor resection with curative intention; 42 (70\%) received concurrent ND, 19 of which $(31.7 \%)$ had pathologic evidence of $\mathrm{N}+$. The median interval from surgery to RT was 30 days. The median dose to the tumor bed was $63 \mathrm{~Gy}$ (range 60-68 Gy). Four patients (6.7\%) received tumor bed irradiation only. Forty-nine (81.7\%) were treated with tumor bed and unilateral neck nodal irradiation, while the rest received tumor bed and bilateral neck nodal irradiation. Four patients received concurrent three-weekly cisplatin chemotherapy. Two received adjuvant chemotherapy after PORT, one with cisplatin and 5-FU and the other with cisplatin and capecitabine. Of the 4 patients who received chemotherapy, 2 exhibited advanced nodal stage (N2b) and 2 major nerve involvement; all presented with stage T3-T4 disease.

\section{Survival}

Kaplan-Meier curves for OS, PFS, LRRFS, and DMFS are shown in Figure 2. The 3-, 5-, and 10-year OS was $90.7 \%, 85.1 \%$, and $85.1 \%$, while that for PFS was $80.1 \%, 72.7 \%$, and $63.1 \%$, respectively. The $3-, 5$, and 10-year LRRFS was $87.4 \%, 82.1 \%$, and $82.1 \%$, while that for DMFS was $85.3 \%, 78.4 \%$, and $66.1 \%$, respectively.

\section{Prognostic factors for OS}

Risk factors for survival are summarized in Table 2\&Additional file 1. Univariable analysis (UVA) suggested that a higher $\mathrm{N}$ stage was associated with decreased survival, with patients having stage N0 disease surviving significantly longer than those with stages $\mathrm{N} 1, \mathrm{~N} 2 \mathrm{~b}$, and $\mathrm{N} 2 \mathrm{c}(p=0.025)$. Primary site was a prognostic factor for OS, with 5-year OS rates of $90 \%, 67.6 \%$, and $100 \%$ for parotid, submandibular, and sublingual gland tumors $(p=0.039)$. However, no significant association was found between OS and $\mathrm{N}$ stage or primary site in MVA.

\section{Prognostic factors for PFS}

Based on our log-rank test, gender was strongly associated with PFS $(p=0.018)$, although it did not reach significance as a predictor in MVA. For patients with and without major nerve involvement, the 5-year PFS was $85 \%$ and $59.5 \%$ ( $p=0.019)$, respectively. Notably, when interval from surgery to RT was analyzed as a categorical variable using the median interval of 30 days as a cutpoint, a significant difference in PFS was observed $(p=0.044)$. No significant difference in PFS was observed among patients with $\mathrm{N}$ stage and positive margin in UVA $(p=0.164,0.092)$. MVA indicated that major nerve involvement (hazard ratio $[\mathrm{HR}]=2.394,95 \%$ confidence interval $[\mathrm{Cl}]=0.664-8.89, p=0.034), \mathrm{N}$ stage $(\mathrm{HR}=0.089,95 \% \mathrm{Cl}=0.008-0.964$, $p=0.047)$, positive margin ( $\mathrm{HR}=4.086,95 \% \mathrm{Cl}=1.097-15.219, p=0.036)$, and interval from surgery to $\mathrm{RT}$ $(\mathrm{HR}=3.934,95 \% \mathrm{Cl}=1.097-14.105, p=0.036)$ were significant independent predictors of PFS (Figure 3$)$. 


\section{Prognostic factors for LRRFS}

In UVA, the 5-year LRRFS for patients with positive margin was $57.1 \%$, compared with $85.9 \%$ for those with negative margin ( $p=0.029)$. In MVA, positive margin was an independent predictor for LRRFS $(\mathrm{HR}=5.064,95 \% \mathrm{Cl}=1.211-21.187, p=0.026)$ (Figure 4).

\section{Prognostic factors for DMFS}

Major nerve involvement, $\mathrm{N}$ stage, and clinical stage were prognostic factors for poor DMFS in UVA $(p=0.024,0.014$, and 0.049 , respectively). When a Cox proportional hazards regression model was used to predict distant metastases, major nerve involvement $(\mathrm{HR}=2.115,95 \% \mathrm{Cl}=0.521-8.583, p=0.008)$, positive margin $(\mathrm{HR}=6.367,95 \% \mathrm{Cl}=1.524-26.603, p=0.011)$, and interval from surgery to $\mathrm{RT}(\mathrm{HR}=6.231,95 \%$ $\mathrm{Cl}=1.503-25.829, p=0.012$ ) were identified as independent predictors of distant metastases (Figure 4).

In ACC subgroup analysis, 5-year OS, PFS, LRRFS, and DMFS were $100 \%, 67.7 \%, 76.2 \%$, and $90.2 \%$, respectively. In UVA, perineural invasion was significantly associated with PFS and DMFS $(p=0.021$ and 0.026 , respectively); major nerve involvement was was strongly associated with PFS ( $p=0.014)$; parotid gland and $\mathrm{N}$ stage were associated with poor LRRFS ( $p=0.041$ and 0.023 , respectively); and positive margin was an important prognostic factor for PFS, LRRFS, and DMFS ( $p=0.021,0.002$, and 0.021 , respectively). However, there were too few ACCs to allow for MVA. For detailed information see Table 3.

\section{Adverse events}

The most acute adverse events were grade $\nabla / \nabla$ mucositis $(n=44,73.3 \%)$ and grade $\nabla / \otimes$ dermatitis $(n=46$, $76.7 \%)$. Xerostomia was the most common late adverse event ( $n=18,30 \%)$, followed by hearing impairment $(n=17,28.4 \%)$, taste abnormalities $(n=15,25 \%)$, paresthesia $(n=14,23.3 \%)$, fibrosis of the skin ( $n=11,18.3 \%)$, trismus $(n=6,10 \%)$, and osteoradionecrosis $(n=2,3.3 \%)$. No grade 4 acute or late adverse events were observed.

\section{Patterns of failure}

Treatment failure occurred in $16(26.7 \%)$ of the 60 patients (Figure 5). Locoregional recurrence occurred in $9(15 \%)$, local failure in 8 (13.3\%), and regional failure in $3(5 \%)$. Distant metastasis occurred in 14 patients $(23.3 \%)$. In the 7 patients with distance-related failure, metastasis was accompanied by locoregional recurrence. The median time to distant metastasis was 49.5 months. Distant metastasis occurred in the lung $(n=10,16.7 \%)$, bones $(n=3,5 \%)$, brain $(n=1,1.7 \%)$, elsewhere $(n=1,1.7 \%)$, and at multiple sites $(n=2,3.3 \%)$.

\section{Discussion}

This retrospective study focused on the clinical outcomes, prognostic factors, failure patterns, and adverse events in patients with major SGC treated with surgery and postoperative IMRT. Similar to that reported in the literature $[3,6,7]$, our study showed that ACC was the most common histology, followed by 
LELC. As histology is an important prognostic factor, for SDC, adenocarcinoma and undifferentiated carcinoma have worse prognosis [8,9], we performed an ACC subgroup analysis. Our clinical cohort exhibited excellent clinical outcomes that compared very favorably with those reported in the literature [10].

Our MVA showed that $\mathrm{N}$ stage, positive margin, major nerve involvement, and interval from surgery to RT were unfavorable prognostic factors. With regard to the relationship between clinicopathologic parameters and OS, there was no negative prognostic factor for survival in MVA. Probable reason was excellent survival of our patients. Although positive margin and $\mathrm{N}$ stage were not prognostic factors for PFS in the UVA, and neither was N stage for LRRFS, we nevertheless included them in the Cox proportional hazards regression model as positive margin and positive lymph nodes were previously shown to be important predictive factors for SGC $[3,11-13]$. After adjusting for factors that might affect prognosis, $\mathrm{N}$ stage and positive margin were found to be independent prognostic factors for PFS, although $\mathrm{N}$ stage was not a predictive factor for LRRFS. Positive margin was also an independent prognostic factor for LRRFS and DMFS, suggesting that margin status information should be included in clinical pathology reports. Major nerve involvement was an independent prognostic factor for poor PFS and DMFS, while a time from surgery to RT $>30$ days resulted in worse PFS and DMFS.

Combination treatment modalities are usually required for SGC. Surgical resection followed by PORT is practical and effective at increasing survival and locoregional control rates in patients with a tumor size $\geq 4 \mathrm{~cm}$, deep lobe settlement, high grade, positive margin, local advanced stage, lymph node metastasis, soft tissue or bone infiltration, and perivascular and perineural invasion [14-17]. Terhaard et al. revealed that the 5- and 10-year actuarial local control rates were significantly higher for PORT vs surgery alone ( $94 \%$ vs $84 \%$ and $91 \%$ vs $76 \%$, respectively) $(p=0.0005)$ [18].

A comparison of our outcomes with those of other institutions is difficult because of different case mix and wide variety of histological subtypes involved. Overall, the outcomes in our study are better than those reported for historical data of other institutions using PORT use for SGC treatment (5-year OS: $85.1 \%$ vs $61-74 \%$; 10 -year OS: $85.1 \%$ vs $48-71 \%$ ) $[15,16,19-21]$. The better survival rates in this series could be explained by a "cohort effect" resulting from improved diagnostic capabilities and treatment modalities. Previous studies have involved conventional RT or 3-DCRT treatment; however, all the patients in this study received IMRT. Compared with conventional RT or 3-DCRT, IMRT permits a greater precision and modulation of the RT beam to keep high doses away from vital structures. This offers improved locoregional control in SGC patients, which corresponds to better OS [22, 23]. Furthermore, because a greater amount of normal tissue is spared, IMRT is less toxic, resulting in a smaller impact on quality of life [24]. Our patients exhibited a reduced incidence of xerostomia (30\%) compared with those from historical studies (83\%) [25].

In agreement with the published literature [26, 27], distant metastasis was the predominant mode of failure, highlighting the need for effective systemic therapies. Chemotherapy is generally reserved for the palliative treatment of symptomatic locally recurrent or metastatic disease, and has a limited effect [28]. 
Recent studies have also indicated that postoperative chemoradiotherapy (POCRT) promotes higher survival and locoregional control than PORT treatment alone [29-32]. However, no significant differences in DMFS, disease-free survival, and OS were observed when adding concurrent chemotherapy to PORT, while POCRT has been associated with increased mortality and toxicity [31,33]. We could not evaluate the effect of POCRT on outcomes owing to the small number of patients who received chemotherapy.

Our study had several significant limitations. The small sample size did permit an evaluation of outcome stratified by various histological subtypes. Additionally, our median follow-up was approximately 55.5 months, and median OS, PFS, LRRFS, and DMFS were not reached. Lastly, The pathological detection of SGC margin status and perineural invasion may vary greatly depending on the extent of sampling. Nevertheless, our observations have potential for use in the design of prospective SGC clinical trials.

\section{Conclusion}

Our series of 60 SGC patients showed that postoperative IMRT leds to improved OS for SGC with acceptable toxicities. $\mathrm{N}$ stage, positive margin, major nerve involvement and interval from surgery to RT were important factors that were associated with oncological outcomes.

\section{Abbreviations}

SGC: salivary gland cancer; IMRT: intensity-modulated radiotherapy; OS: overall survival; PFS: progression-free survival; LRRFS: locoregional relapse-free survival; DMFS: distant metastasis-free survival; ACC: adenoid cystic carcinoma; MVA: multivariable analysis; RT: radiotherapy; PORT: postoperative radiotherapy; UVA: Univariable analysis; POCRT: postoperative chemoradiotherapy; CT: computed tomography; MRI: magnetic resonance imaging; PET: positron emission tomography; ND: neck dissection; CTV: Clinical target volume; PTV: planning target volume; LELC: lymphoepithelial carcinoma; MEC: mucoepidermoid carcinoma; SDC: salivary duct carcinoma; SCC: squamous cell carcinoma; BCAC: basal cell adenocarcinoma; AcCC: acinic cell carcinoma; POCRT: postoperative chemoradiotherapy

\section{Declarations}

\section{Ethics approval and consent to participate}

For this type of study ethics approval is not required. Written informed consent was obtained 'front door' from all patients allowing collection of their clinical data.

\section{Consent for publication}

Not applicable. The MRI images displayed in the paper have been anonymized, and cannot be used to identify patients.

\section{Availability of data and materials}


The datasets generated and/ or analysed during the current study are not publicly available due [REASON WHY DATA ARE NOT PUBLIC] but are available from the corresponding author on reasonable request.

\section{Conflicts of interest}

There are no conflicts to declare.

\section{Funding}

This study was independent research funded by the Department of Health of Zhejiang Province (2018265493, 2017197137).

Authors' contributions

Danfang Yan and Senxiang Yan designed this study; Senxiang Yan also revised the main manuscript content; Shoumei Zang and Huijie Huang carried out analysis and wrote the manuscript; Xinli Zhu and Meiqin Chen were responsible for data collection.

\section{Acknowledgements}

Not applicable.

\section{References}

[1] Ata-Ali J, Zurriaga O, Alberich C. Incidence and survival rates for malignant salivary gland tumors. J Oral Sci 2016;58:67-73.

[2] Spiro RH. Salivary neoplasms: overview of a 35-year experience with 2,807 patients. Head Neck Surg 1986;8:177-84.

[3] Terhaard CH, Lubsen H, Van der Tweel I, et al. Salivary gland carcinoma: independent prognostic factors for locoregional control, distant metastases, and overall survival: results of the Dutch head and neck oncology cooperative group. Head Neck 2004;26:681-92; discussion 692-3.

[4] Lima RA, Tavares MR, Dias FL, et al. Clinical prognostic factors in malignant parotid gland tumors. Otolaryngol Head Neck Surg 2005;133:702-8.

[5] Thompson L. World Health Organization classification of tumours: pathology and genetics of head and neck tumours. Ear Nose Throat J 2006;85:74

[6] Bell RB, Dierks EJ, Homer L, Potter BE. Management and outcome of patients with malignant salivary gland tumors. J Oral Maxillofac Surg 2005;63:917-28.

[7] Kaur J, Goyal S, Muzumder S, Bhasker S, Mohanti BK, Rath GK. Outcome of surgery and post-operative radiotherapy for major salivary gland carcinoma: ten year experience from a single institute. Asian Pac $J$ 
Cancer Prev 2014;15:8259-63.

[8] Wahlberg P, Anderson H, Biorklund A, Moller T, Perfekt R. Carcinoma of the parotid and submandibular glands-a study of survival in 2465 patients. Oral Oncol 2002;38:706-13.

[9] Jang JY, Choi N, Ko YH, et al. Treatment outcomes in metastatic and localized high-grade salivary gland cancer: high chance of cure with surgery and post-operative radiation in T1-2 N0 high-grade salivary gland cancer. BMC Cancer 2018;18:672.

[10] Ali S, Palmer FL, Katabi N, et al. Long-term local control rates of patients with adenoid cystic carcinoma of the head and neck managed by surgery and postoperative radiation. Laryngoscope 2017;127:2265-9.

[11] Qian K, Di L, Guo K, Zheng X, Ji Q, Wang Z. Cervical Lymph Node Metastatic Status and Adjuvant Therapy Predict the Prognosis of Salivary Duct Carcinoma. J Oral Maxillofac Surg 2018;76:1578-86.

[12] Yoo SH, Roh JL, Kim SO, et al. Patterns and treatment of neck metastases in patients with salivary gland cancers. J Surg Oncol 2015;111:1000-6.

[13] Johnston ML, Huang SH, Waldron JN, et al. Salivary duct carcinoma: Treatment, outcomes, and patterns of failure. Head Neck 2016;38 Suppl 1:E820-6.

[14] Cerda T, Sun XS, Vignot S, et al. A rationale for chemoradiation (vs radiotherapy) in salivary gland cancers? On behalf of the REFCOR (French rare head and neck cancer network). Crit Rev Oncol Hematol 2014;91:142-58.

[15] Storey MR, Garden AS, Morrison WH, Eicher SA, Schechter NR, Ang KK. Postoperative radiotherapy for malignant tumors of the submandibular gland. Int J Radiat Oncol Biol Phys 2001;51:952-8.

[16] North CA, Lee DJ, Piantadosi S, Zahurak M, Johns ME. Carcinoma of the major salivary glands treated by surgery or surgery plus postoperative radiotherapy. Int J Radiat Oncol Biol Phys 1990;18:131926.

[17] Pohar S, Gay H, Rosenbaum P, et al. Malignant parotid tumors: presentation, clinical/pathologic prognostic factors, and treatment outcomes. Int J Radiat Oncol Biol Phys 2005;61:112-8.

[18] Terhaard $\mathrm{CH}$, Lubsen $\mathrm{H}$, Rasch CR, et al. The role of radiotherapy in the treatment of malignant salivary gland tumors. Int J Radiat Oncol Biol Phys 2005;61:103-11.

[19] Toonkel LM, Guha S, Foster P, Dembrow V. Radiotherapy for parotid cancer. Ann Surg Oncol 1994;1:468-72.

[20] Fitzpatrick PJ, Theriault C. Malignant salivary gland tumors. Int J Radiat Oncol Biol Phys 1986;12:1743-7. 
[21] Bhide SA, Miah A, Barbachano Y, Harrington KJ, Newbold K, Nutting CM. Radical radiotherapy for treatment of malignant parotid tumours: a single centre experience. 1995-2005. Br J Oral Maxillofac Surg 2009;47:284-9.

[22] Chen JL, Huang YS, Kuo SH, et al. Intensity-modulated radiation therapy achieves better local control compared to three-dimensional conformal radiation therapy for T4-stage nasopharyngeal carcinoma. Oncotarget 2017;8:14068-77.

[23] Hosni A, Huang SH, Goldstein D, et al. Outcomes and prognostic factors for major salivary gland carcinoma following postoperative radiotherapy. Oral Oncol 2016;54:75-80.

[24] van der Veen J, Nuyts S. Can Intensity-Modulated-Radiotherapy Reduce Toxicity in Head and Neck Squamous Cell Carcinoma? Cancers. 2017;9(10). pii: E135.

[25] Nutting CM, Morden JP, Harrington KJ, et al. Parotid-sparing intensity modulated versus conventional radiotherapy in head and neck cancer (PARSPORT): a phase 3 multicentre randomised controlled trial. Lancet Oncol 2011;12:127-36.

[26] Samant S, van den Brekel MW, Kies MS, et al. Concurrent chemoradiation for adenoid cystic carcinoma of the head and neck. Head Neck 2012;34:1263-8.

[27] Hosal AS, Fan C, Barnes L, Myers EN. Salivary duct carcinoma. Otolaryngol Head Neck Surg 2003;129:720-5.

[28] Vander Poorten VL, Balm AJ, Hilgers FJ, Tan IB, Keus RB, Hart AA. Stage as major long term outcome predictor in minor salivary gland carcinoma. Cancer 2000;89:1195-204.

[29] Tanvetyanon T, Qin D, Padhya T, et al. Outcomes of postoperative concurrent chemoradiotherapy for locally advanced major salivary gland carcinoma. Arch Otolaryngol Head Neck Surg 2009;135:687-92.

[30] Maruya S, Namba A, Matsubara A, et al. Salivary gland carcinoma treated with concomitant chemoradiation with intraarterial cisplatin and docetaxel. Int J Clin Oncol 2006;11:403-6.

[31] Hsieh CE, Lin CY, Lee LY, et al. Adding concurrent chemotherapy to postoperative radiotherapy improves locoregional control but not overall survival in patients with salivary gland adenoid cystic carcinoma-a propensity score matched study. Radiat Oncol 2016;11:47.

[32] Schoenfeld JD, Sher DJ, Norris CM, et al. Salivary gland tumors treated with adjuvant intensitymodulated radiotherapy with or without concurrent chemotherapy. Int J Radiat Oncol Biol Phys 2012;82:308-14.

[33] Tanvetyanon T, Fisher K, Caudell J, Otto K, Padhya T, Trotti A. Adjuvant chemoradiotherapy versus with radiotherapy alone for locally advanced salivary gland carcinoma among older patients. Head Neck 2016;38:863-70. 


\section{Tables}

Due to technical limitations, the tables are only available as a download in the supplemental files section.

\section{Figures}
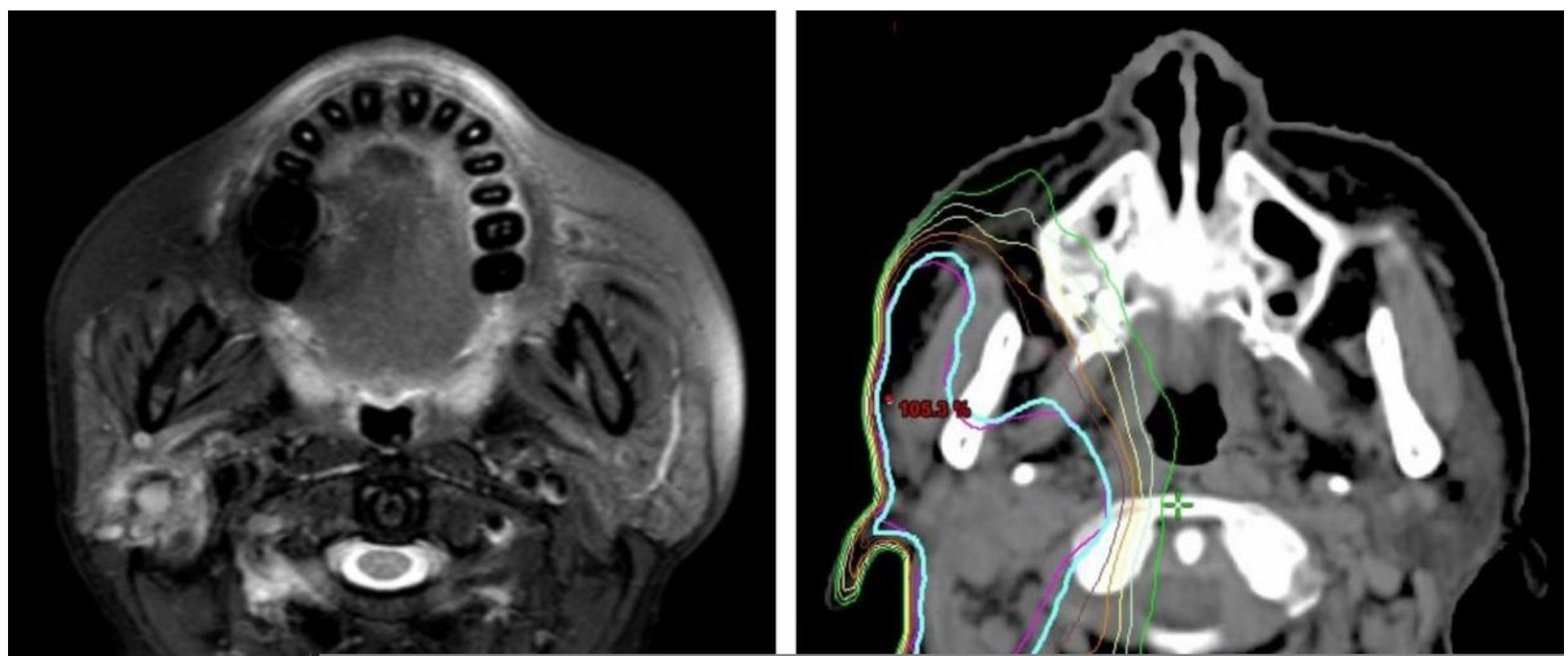

\section{Figure 1}

A typical case of right parotid gland tumor (a) Axial T2-weighted magnetic resonance imaging (MRI) (b) Isodose line of treatment plans of intensity modulated radiotherapy (IMRT)

\section{Figure 2}

Kaplan-Meier curves of overall survival (OS), progression-free survival (PFS), locoregional relapse-free survival (LRRFS), distant metastasis-free survival (DMFS) 
a

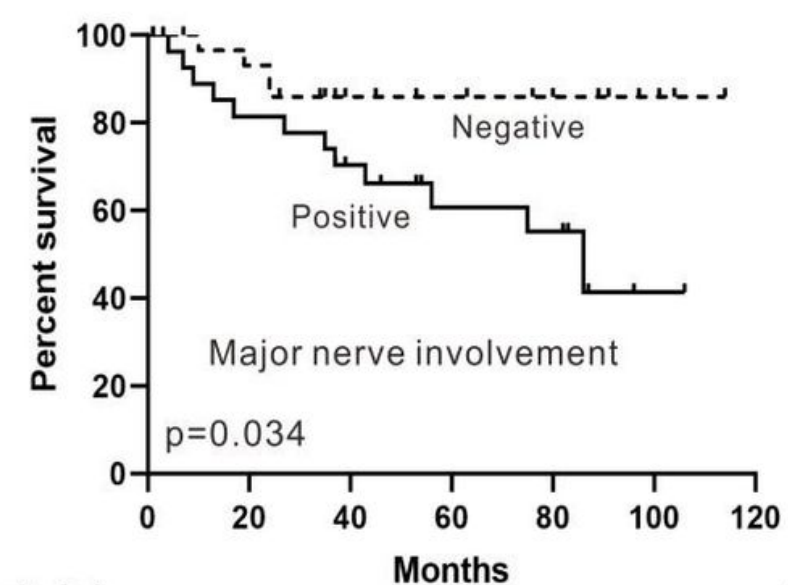

b

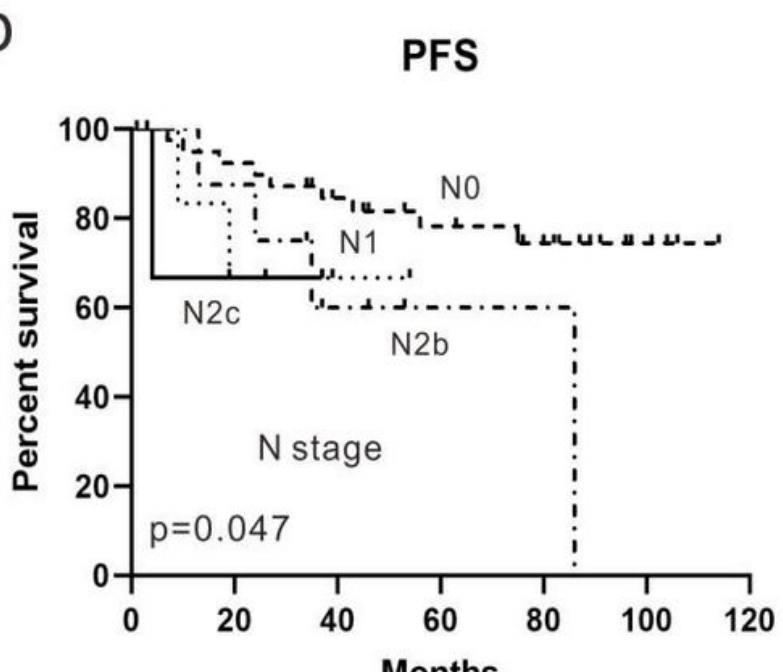

Patients at risk

\section{Patients at risk}

$\begin{array}{cccccccc}\text { Positive } & 28 & 21 & 17 & 13 & 12 & 2 & 1 \\ \text { Negative } & 32 & 29 & 18 & 13 & 9 & 4 & 1\end{array}$

C

PFS

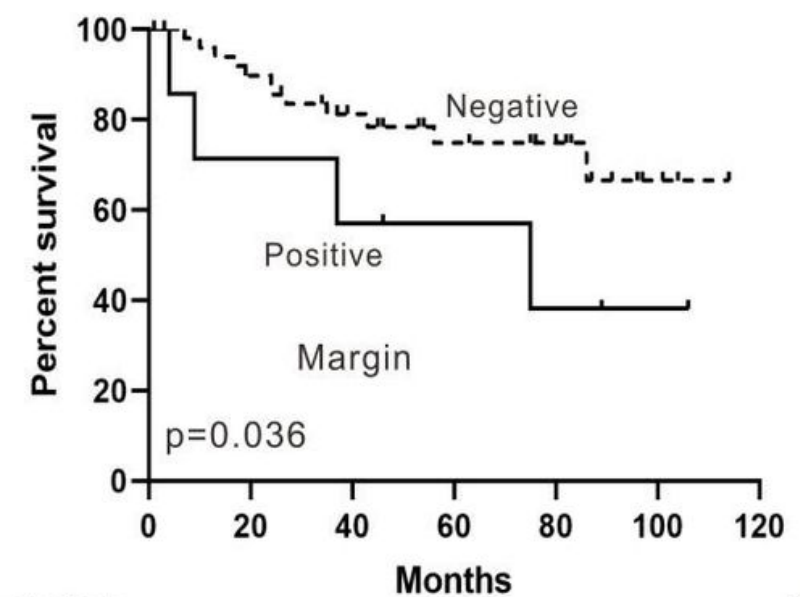

$\begin{array}{llllllll}\text { No } & 41 & 37 & 30 & 24 & 18 & 6 & 1\end{array}$

$\begin{array}{llllllll}\mathrm{N} 1 & 8 & 5 & 2 & 1 & 1 & 1 & 1\end{array}$

$\begin{array}{llllllll}\mathrm{N} 2 \mathrm{~b} & 8 & 8 & 4 & 2 & 2 & 1 & 1\end{array}$

$\begin{array}{llllllll}\mathrm{N} 2 \mathrm{C} & 3 & 2 & 1 & 1 & 1 & 1 & 1\end{array}$

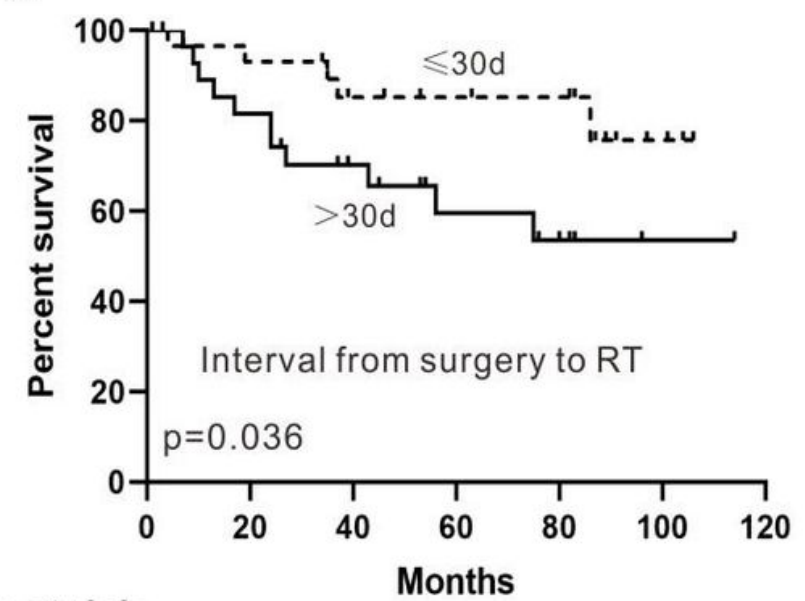

d

\section{PFS}

$\begin{array}{lllllcll}\leqslant 30 & 30 & 28 & 19 & 16 & 14 & 5 & 1 \\ >30 & 30 & 23 & 16 & 11 & 7 & 2 & 1\end{array}$

\section{Patients at risk}

$\begin{array}{llllllll}\text { Positive } & 7 & 6 & 5 & 4 & 3 & 2 & 1\end{array}$

\section{Patients at risk}

\section{Figure 3}

Comparison of survival according to clinicopathologic factors. (a) N stage $(p=0.047)$; (b) margin $(p=0.036) ;(c)$ major nerve involvement $(p=0.034)$, (d) interval from surgery to RT $(p=0.036)$ 
a

\section{LRRFS}

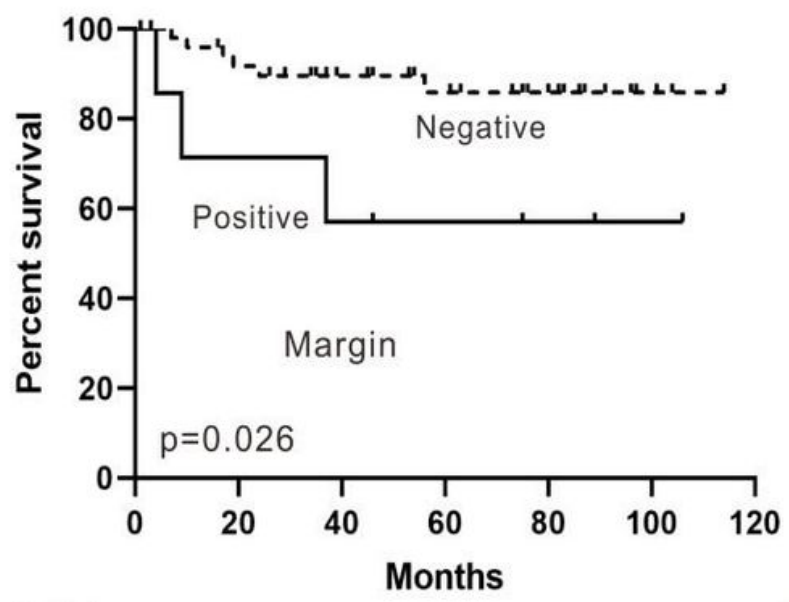

b

DMFS

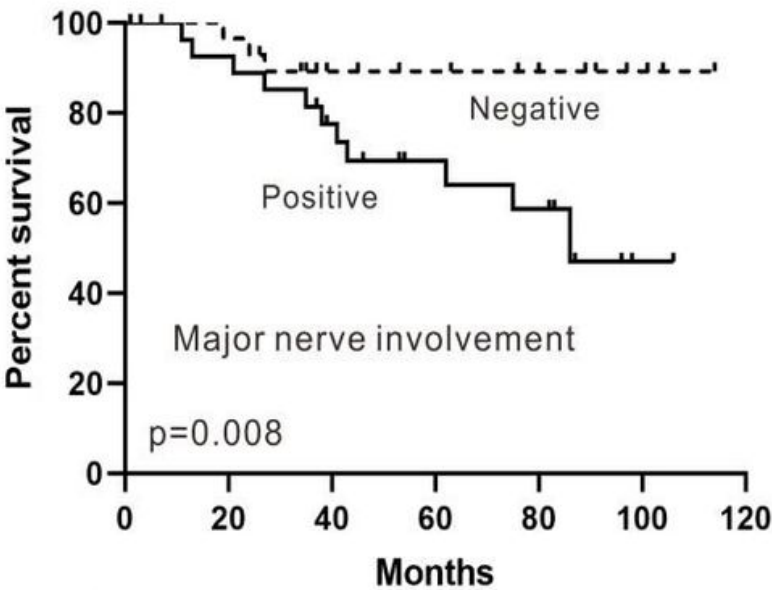

\section{Patients at risk}

\section{Patients at risk}

$\begin{array}{llllllll}\text { Positive } & 7 & 6 & 5 & 4 & 3 & 2 & 1\end{array}$

$\begin{array}{llllllll}\text { Negative } & 53 & 45 & 32 & 24 & 17 & 5 & 1\end{array}$

$\begin{array}{llllllll}\text { Positive } & 28 & 25 & 19 & 14 & 12 & 2 & 1 \\ \text { Negative } & 32 & 29 & 17 & 15 & 10 & 5 & 1\end{array}$

C

DMFS

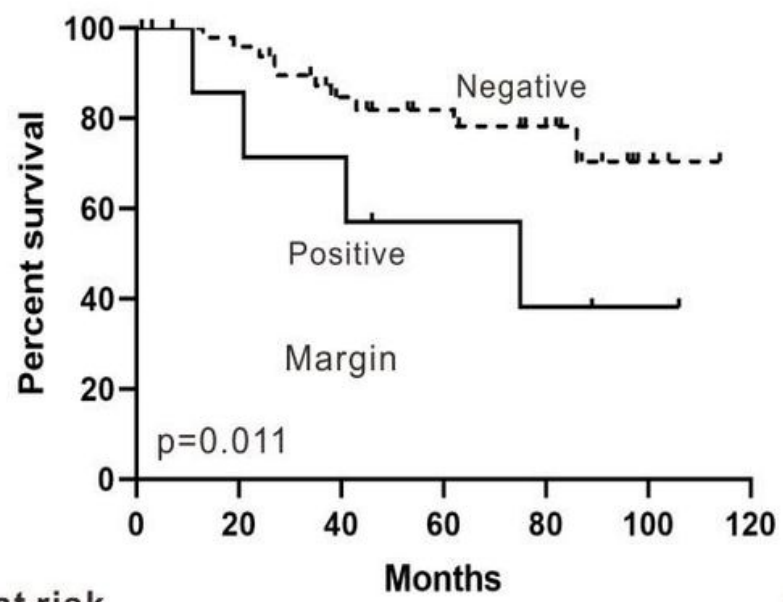

\section{Patients at risk}

$\begin{array}{cccccccc}\text { Positive } & 7 & 7 & 6 & 4 & 3 & 2 & 1 \\ \text { Negative } & 53 & 48 & 32 & 24 & 18 & 4 & 1\end{array}$

d

DMFS

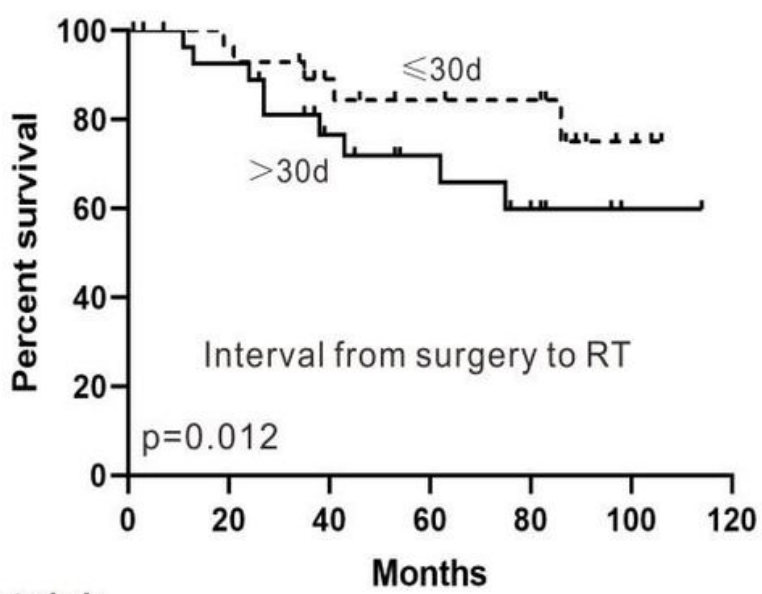

\section{Patients at risk}

$\begin{array}{lllllcll}\leqslant 30 & 30 & 29 & 20 & 16 & 14 & 5 & 1 \\ >30 & 30 & 26 & 17 & 13 & 8 & 2 & 1\end{array}$

\section{Figure 4}

Comparison of survival according to clinicopathologic factors. (a) margin ( $p=0.026)$; (b) major nerve involvement $(p=0.008) ;(c)$ margin $(p=0.011)$; $(d)$ interval from surgery to RT $(p=0.012)$

Figure 5

Venn diagram of patterns of failure (16/60 patients) 


\section{Supplementary Files}

This is a list of supplementary files associated with this preprint. Click to download.

- Tables.pdf

- Additionalfile1.pdf 